\title{
A FIREFLY OPTIMIZED LSTM RISK DETECTION \& PREDICTION MODEL FOR IOT ENABLED SUPER MARKETS
}

\author{
RR Karthikeyan \\ Research Scholar, Bharath Institute of Higher Education and Research, Chennai \\ rrkarthikeyan0711@gmail.com \\ Dr. B. Raghu \\ Principal of SVS Group of Institutions, Warangal, Telangana India \\ raghubalraj@gmail.com
}

\begin{abstract}
The worldwide network has to a great extent perceived that the Earth's atmosphere is evolved a lot in the last few decades. In particular, the Climatic Action attempts to both reduction of European Union (EU) ozone harming substance outflows and enhance the efficiency by decreasing the consumption of essential energy. In Retail shops and commercial buildings are liable to consistently monitor and control for the Heating, Ventilation, and Air Conditioning (HVAC) and refrigeration systems. As per the monstrous Internet Of thigh's (IoT) collection of data, there are unnecessary utilization of energy may happen because of manual activity in the Retail shops and commercial buildings. Recent decades, smart supermarkets are implemented by tuning HVAC systems and the refrigeration system automatically for the purpose of improving the satisfaction of customers and also optimizing energy consumption. To achieve an agenda, in this paper, it plans to build up a technique for (1) investigating the sensor model depending on the detected data; (2) constructing a forecasting model for a working status of systems, and proposing a Firefly based optimized Long Short-Term Memory Network (FOLSTM) model for the advance forecasting of data; (3) improving the forecast precision utilizing FOLSTM with the comparison of conventional methods. This FOLSTM technique with real-time collected data from the sensors of an HVAC and the refrigeration framework where the information is appropriate to general IoT hardware for investigating the accuracy and the determining prediction status.
\end{abstract}

Keywords: LSTM Prediction; IoT Enabled Supermarket; HVAC and refrigeration systems.

\section{Introduction}

Since the Industrial Revolution, the improvement of innovations, and the development of populace have expanded energy utilization around the globe. This causes ecological issues, for example, consumption of energy assets, a worldwide temperature alteration, and environmental variations. Energy utilization is expanding in a worldwide step by step, because of the limited energy assets, the energy issue has become a hotly debated issue everywhere on the world with effort for alterations. Recent time's supper business sectors are expanding, and warming and cooling, lighting, and ventilation frameworks are progressively utilized. This is required to expand the measure of energy utilized in structures. Considering supermarket zone, energy utilized in Refrigeration and HVAC frameworks make up an enormous piece of building energy utilization, the financial effect that can be picked up by decreasing the energy utilized is huge.

The EU has highlighted the advancement of efficient energy management that the Supermarkets do have extremely high energy utilization, of $300-600 \mathrm{kWh} / \mathrm{m} 2$, which is contrasted with other business (places of business $\sim 150-200 \mathrm{kWh} / \mathrm{m} 2$ ) Improvements will accordingly give critical savings of energy. However, it is a difficult task that has a numerous sensors are included such as temperature, pressure, occupancy, indoor air quality, compressor control, building envelope, time of shop opening, presence of an individual's etc. Most of the energy is generated by HVAC frameworks that have solid effect comfort just as on the climate.

In olden retail shops and buildings, the clients are responsible for observing and controlling gadget's availability. Nonetheless, present-day smart shops are progressively equipped with IoT gadgets and hardware like actuators, sensors, heaters and air control systems. In such shops, and IoT gadgets team up to change temperature, pressure and optimize the HVAC utilization and in refrigeration by predicting the indoor temperature, object's occupancy and pressure and also tuning HVAC and refrigerant gadgets for predicting the streamline of energy utilization.

In this work, an agenda is to propose a firefly based optimized LSTM (FOLSTM) techniques for improving the system's prediction level and performance which are incorporated into building management systems (BMS) for 
enhancing efficiency. IN particular, HVAC and refrigeration framework are considered with a customer's comfort levels and also reducing the usage of energy with the accurate problem forecasting.

The rest of the article is arranged as follows. In section 2, the sensor is utilized in the HVAC and the refrigeration is described. Section 3 illustrated the existing forecasting of energy in different buildings and shops. In section 4, requirement and the set points of supermarket design is explained and the proposed methodology is described with its preliminaries in section 5. Section 6 contains the results of proposed techniques with the comparisons. Finally, the paper is concluded in section 7.

\section{Different types of Sensors in HVAC and Refrigeration System}

In this section, the sensors are used in the HVAC systems and refrigerators are mentioned. The following sensors are considered as important parameters in this proposed model where the sensed data are collected from it.

In the HVAC system, the following sensors are used for smoothness and safety to enhance the energy efficiency, and also for the convenience of human.

Pressure sensors are utilized for screening the shops and filtering for drops in pressure that indicate the system's maintenance. The pressure value is significant for optimizing airflow, cooling and heating.

Duct smoke detector is utilized to prevent the building from spreading the smoke. It could be needed by guidelines and codes of building.

Occupancy sensors are utilized to distinguish the presence of a person in a shop. It can enable clients to improve their energy proficiency. It has two main types namely PIR sensor and ultra-sonic sensor. The PIR sensor is utilized to quantify heat and movement. Then Ultrasonic sensors detect objects that can go through an object's strong which is more modest and sensitive than PIR sensors.

Indoor air quality (IAQ) is a carbon sensor that distinguishes carbon levels noticeable all around in air. Carbon dioxide sensors utilize either infrared light or a process of chemical for measuring a level of $\mathrm{CO} 2$. It is additionally used to identify volatile organic compounds (VOCs) which can ionize particles with bright light and measure electron levels.

In the Refrigeration system, there are three important controls are used in it namely evaporator control, condenser control and compressor control.

Evaporator Control is a control that is utilized to detect an evaporator's temperature and pressure by sensors. It likewise naturally regulate the refrigerant flow through the evaporator to keep up the refrigerated space inside precharacterized working cutoff limits.

Condenser Control is utilized to detect a condenser temperature and pressure by sensors. It naturally regulates the flow of air over the condenser. To keep up the system's thermal balance under various working and climate conditions, this maintains the condensation rate requirements.

Compressor Control has programmed to screen the suction's pressure or refrigerant temperature. It is utilized to keep it away from superfluously being controlled to load or empty in light of little fluctuations in cooling interest.

\section{Related Works}

In this section, the previous studies have established the energy prediction in HVAC and refrigeration which consumes the huge energy in a supermarket and all other buildings. Therefore, the related papers have a qualified effect on both user comfort and energy consumption forecasting in earlier stage.

Kwok et al (2011) adjusted the cooling load by fusing a Neural Network (NN) into a design that permits prediction and analysing the required energy as well as for deciding the basic factors those impacts on the utilization of energy. Nguyen et al (2013) examined the effect of the two clients' exercises and their practices on saving of potential energy in smart shop. This paper separated the client on energy requests impact into three primary subsystems such as HVAC, plug load and light system. Another model of Support Vector Regression (SVR) is presented to foresee the cooling load in shops by Li et al (2009). The hyper-boundaries were used for estimating the best temperature. Xu et al (2019) presented an LSTM model to predict the temperature earlier of 5 and 30 min. It is compared with Decision Tree, Back Propagation Neural Network, and Support Vector Machine which gives the better result than the existing. Abdullatif et al (2004) developed a predictive model of cooling load by using a generalized regression neural network (GRNN). The main considerations are structure orientation characteristics and occupancy to optimize the HVAC's energy consumption. The implementation of the decision support framework is described by Doukas et al (2007) which are used to enhance the energy by allowing the control of central for the enormously flexible. 
Sadi Alawadi et al (2020) presented forecasting of indoor temperatures with the comparison of several machine learning systems. This paper compares the 36 Machine Learning techniques of HVAC and refrigeration system using real data that improves accuracy respectively. The algorithm of the high-efficiency motor to an HVAC and refrigeration system is described using a large number of motors by Dezfouli et al (2018). It has experimented that the high-efficiency motors can be achieved energy savings. Long Chang et al (2017) described a classification algorithm using a cloud for the purpose of efficient fault recognition and analysis of HVAC and refrigeration systems. Preglej et al (2014) implemented a fuzzy method on multivariate forecasting to control the system. This model is presented in the actual test site which is resultant in the reduction of energy consumption. Beghi et al (2010) described forecasting the load of cooling in systems based on artificial neural network (ANN). By using this model, there is a lot of research has done to store energy consumption in various features.

\section{Supermarket Requirements}

In this section, the energy consumption of supermarkets is explained in order to save energy and also explains the parameter that is influencing energy consumption. The supermarket building is categorised into few subsystems as shown Figure1. In the poppi (2010) case study, a $2125 \mathrm{~m} 2$ supermarket which is a single floor constructing north-south situated with $19,8 \mathrm{~m} 2$ windows. The shop is thought to be open from 8 am to $10 \mathrm{pm}$ from Monday to Saturday. The area of shop incorporates show cupboards for chilled (MT) and solidified (LT) items, dry merchandise racks and cold rooms. Insights about cooling loads for cupboards and cold rooms are summed up in Table 1.

Table 1: Cooling load for cabinets and cold rooms

\begin{tabular}{|l|l|l|l|l|l|l|}
\hline & $\begin{array}{l}\text { MT vertical } \\
\text { multi deck }\end{array}$ & $\begin{array}{l}\text { MT } \\
\text { Open island }\end{array}$ & $\begin{array}{l}\text { MT } \\
\text { Semi- } \\
\text { vertical }\end{array}$ & $\begin{array}{l}\text { LT open } \\
\text { Wall site }\end{array}$ & $\begin{array}{l}\text { MT } \\
\text { Cold room } \\
+2{ }^{\circ} \mathrm{c}\end{array}$ & $\begin{array}{l}\text { LT } \\
\text { Cold room } \\
-22^{\circ} \mathrm{c}\end{array}$ \\
\cline { 2 - 7 } & Rack system & $\begin{array}{l}\text { Rack } \\
\text { system }\end{array}$ & $\begin{array}{l}\text { Rack } \\
\text { system }\end{array}$ & $\begin{array}{l}\text { Plug in } \\
\text { units }\end{array}$ & $\begin{array}{l}\text { Rack } \\
\text { system }\end{array}$ & $\begin{array}{l}\text { Rack } \\
\text { system }\end{array}$ \\
\hline $\begin{array}{l}\text { Evap } \\
\text { temp }{ }^{\circ} \mathrm{C}\end{array}$ & -10 & -10 & -10 & -35 & -10 & -35 \\
\hline $\begin{array}{l}\text { Nominal } \\
\text { HER-W/m }\end{array}$ & 1028 & 400 & 2263 & 845 & & \\
\hline $\begin{array}{l}\text { Total length- } \\
\text { m }\end{array}$ & 3335 & 225 & 68 & 12 & & \\
\hline $\begin{array}{l}\text { Total HER- } \\
\text { W }\end{array}$ & 34283 & 9000 & 15388 & 10140 & 10411 & 2633 \\
\hline
\end{tabular}

\subsection{Supermarket Sections}

\section{Climate of Outdoor}

For the structure thermal load calculation, the parameters like air temperature, humidity, wind speed, radiation of the sun, sun's height, and sun based azimuth are required.

\section{Building}

The necessary building structure envelope of heating and cooling is given in this part. It considers convection through the structure surfaces (dividers, rooftop and ceiling) air invasions and inward warmth loads, for example, reasonable and latent warmth from individuals, lighting and other electrical burdens, cupboards and cold rooms. Every individual's Presence in the shop has been accepted and changed for the genuine logged normal absolute number of clients during a day.

\section{Indoor climate}

The threshold of day-night time is similar to the outside temperature to enhance the efficiency of the system. The indoor air temperature is $21^{\circ} \mathrm{C}$ at lower surrounding temperature, yet is permitted to increment if ambient temperature increments during summer. When ambient is higher than $27^{\circ} \mathrm{C}$ then the maximum indoor temperature is $25^{\circ} \mathrm{C}$. The overall moistness in the shop has been set to $55 \%$. Air quality relies upon the convergence of carbon dioxide in the shop. Estimations show that $\sim 2.5310-6 \mathrm{~m} 3 / \mathrm{s}$ per individual of outside air is expected to keep the maximum concentration of $1000 \mathrm{ppm}$ consistent.

\section{Refrigeration system}

The refrigeration is presently introduced in the general store is a CO2 DX course framework. The rack of the compressor has been measured utilizing the absolute cooling load mentioned by cupboards and cold rooms: 77,5 and 2,6 kW individually for MT and LT applications, module units excluded. The pressure in evaporator has been 
fixed at 26 bar for MT utilities and 12 bar for LT utilities. The heat exchangers have been considered in cupboards and cold rooms.

The refrigeration side has been determined considering isothermal conditions with $5 \mathrm{~K}$ superheating at the evaporator and no subcooling at the condenser. The build-up of the LT circuit happens in a heat exchanger's plate; $-7^{\circ} \mathrm{C}$ is the consolidating temperature. Abundance heat is dismissed in an air cooler, going about as a gas cooler or air-cooled condenser relying upon the working conditions. The gas cooler optimised weight follows the saturation bend until $20^{\circ} \mathrm{C}$ source temperature and afterwards a direct pattern has been fixed.

When most extreme heat load has been mentioned, then the Air cooler condenser and gas cooler are estimated. In at condenser (temperature distinction between ambient air and temperature) and gas cooler methodology in these conditions has $20 \mathrm{~K}$ and $10 \mathrm{~K}$ have been accepted individually as. At the point when the lower heat load is required, it has been diminished.

As indicated by the external atmosphere where the input signal is gotten from the air temperature sensor, control framework deals with the 3-way plug valve: bypass is actuated when air temperature is lower than a fixed set.

The absolute warmth request can be furnished by the supporter refrigeration rack with the dismissal of indirect heat: water is shipped off the floor warming circuits and to the warmth exchangers of the HVAC unit. At the point when extra cooling is required, MT function as a reversible heat pump and produce heat.

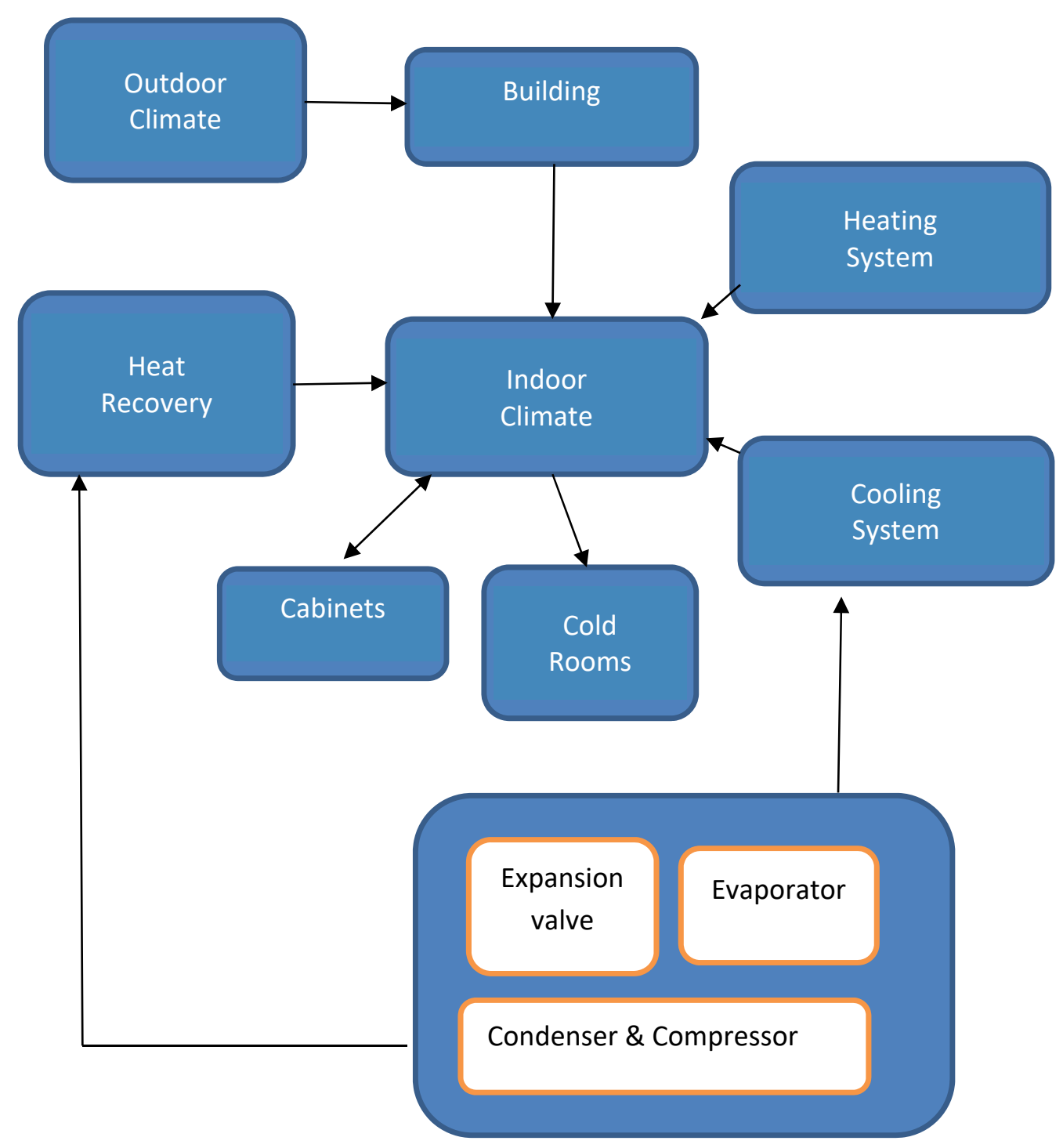

Fig. 1. Supermarket Sections 


\section{PROPOSED SYSTEM}

In this section, the investigation of HVAC and the refrigeration system are depending on the sensor models to detect the data of temperature, pressure, and the control units. The information sets have been downloaded from METEONORM. METEONORM utilizes the normal qualities from the period 1961-1990 for various climate stations as per the World Meteorological Institute (WMO) atmosphere typical (Remund 2005). The Forecasting model is designed by combining a two algorithm namely firefly optimisation algorithm, and LSTM techniques which are termed as Firefly based optimized Long Short-Term Memory Network (FOLSTM) model. Heretofore, firefly based heuristics used to find the time window size and architectural LSTM's hyperparameter factors. By using FOLSTM model, the prediction is improved efficiently which is compared with the conventional methods.

\section{1 preliminaries}

\section{Firefly Optimization Algorithm (FOA)}

A firefly optimization algorithm was a meta- heuristic algorithm which is motivated by a lifestyle of fireflies. The normal behavior of firefly was creating rhythmic and short blazes which is created by a process of bioluminescence according to inverse square law. The Fireflies are utilizing their flashes light for hunting, communication and cautioning their enemies. consider Light power (I) at a particular distance (r) where the light power diminishes with the increments of the distance between the fireflies. Also, the light and its power diminishes by air absorption and gets more vulnerable as distance increments. Hence, most fireflies communicate within a hundred meters distances which were its usual limit. Hence, the objective function is the blazing light which derived a population-based Firefly algorithm.

The light power $\mathrm{I}(r)$ ) at $\mathrm{r}$ distance from a light source (ls ) is can be determined using inverse square law which is given below.

$$
I(r)=l_{s} / r^{2}
$$

The light is immersed in the air with a constant coefficient of light absorption $(\gamma \in(0, \infty)$. Therefore the Gaussian equation is formed and given in the following by Equation (2)

$$
B(r)=B_{0} e^{-\gamma r^{2}}
$$

Where $(r)$ is fireflies attractiveness at distance $r$ and when $r=0$, then $B_{0}$ is attractiveness.

Consider two fireflies $i$ and $j$ with its positions $\left(, y_{i}\right)$ and $X_{j}\left(x_{j}, y_{j}\right) . r_{i j}$ is the two fireflies distance and the formulation is expressed by Euclidean which is shown in Equation (3)

$$
r_{i j}=\left\|X_{i}-X_{j}\right\|=\sqrt{\left(x_{i}-x_{j}\right)^{2}-\left(y_{i}-y_{j}\right)^{2}}
$$

The new position ( ) of brighter firefly and its movement $(j)$ is expressed in the following.

$X_{i}=X_{i}+B_{0} e^{-\gamma r^{2}}\left(X_{j}-X_{i}\right)+a \epsilon_{i}$

Where $\epsilon_{i}$ is random vector variables and also the parameter of randomness $(\alpha) \in[0,1]$.

\section{Long Short-Term Memory (LSTM)}

Jürgen Schmid Huber proposed the LSTM dependent on the guideline of recurrent networks, the feedback connections stored the current info functions in the structure of the initiation (short-term) memory. Notwithstanding, error signals that were owing in reverse in time will in general explode or disappear. The cell state is the LSTM's key, which fills in as a transport line. The cell state directly runs the whole chain, with just some minor cooperations linearly. The gate structures are applied and used for regulations, LSTM can eliminate or add data to the phone state. Subsequently, LSTM was purposely designed to prevent dependency issues in long terms. There are three gates are presented in every cell such as input gate, output gate and forget gate. Assign an input of $\mathrm{x}(\mathrm{t})$ and $\mathrm{h}(\mathrm{t}-1)$ and then every cell computation are expressed in the following.

$$
\begin{aligned}
& f_{t}=\sigma\left(w_{f} \cdot\left[h_{t-1}, x_{t}\right]+b_{f}\right) \\
& i_{t}=\sigma\left(w_{i} \cdot\left[h_{t-1}, x_{t}\right]+b_{i}\right) \\
& g_{t}=\tanh \left(w_{g} \cdot\left[h_{t-1}, x_{t}\right]+b_{g}\right) \\
& o_{t}=\sigma\left(w_{o} \cdot\left[h_{t-1}, x_{t}\right]+b_{o}\right) \\
& \left.C_{t}=f_{t} \cdot C_{t-1}+g_{t} \cdot i_{t}\right) \\
& Y_{t}=\tanh \left(f_{t} \cdot\left[C_{t-1}+g_{t} \cdot i_{t}\right] \cdot o_{t}\right)
\end{aligned}
$$


Where the $t$ indicates the timestamp, $\mathrm{C}_{\mathrm{t}}$ is the cell state and $\mathrm{y}_{\mathrm{t}}$ is the output and it acts as a next timestamp input. The gates output are $f_{t}, i_{t}, g_{t}, o_{t}$ and the coefficient matrixes are $W_{i}, W_{f}, W_{o}, W_{g}, b_{i}, b_{f}, b_{o}$ and $b_{g}$ respectively.

The data are corrupted by the error with the different cells interactions of input and output. Thus, the LSTM's loss function $(\mathrm{L}(t))$ has two blocks namely loss at the time $t$, and the spread back loss from timestamp after the time $t$ :

$$
L(t)= \begin{cases}l(t)+L(t+1) & t<\tau \\ l(t) & t=\tau\end{cases}
$$

Where $\tau$ is the last timestamp index.

\subsection{Proposed methodology cc}

\section{FOLSTM TECHNIQUE}

In general, LSTM utilizes previous data during the learning process, a proper selection of time window plays a significant role in an assured performance. The training model will omit important data when the window is too small.

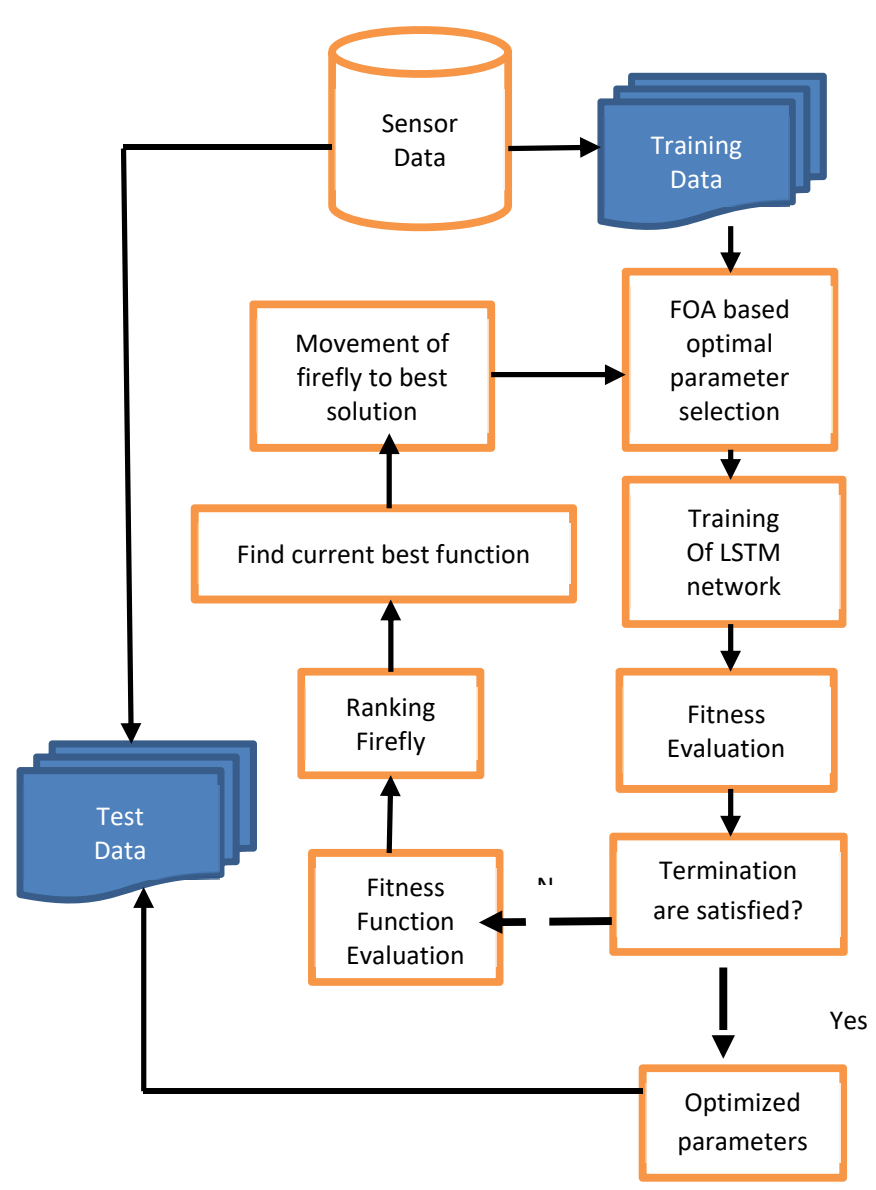

Fig. 2. Proposed prediction model.

Similarly, the model over fitted with training data when the window size is too big. The proposed prediction includes two stages, the proper selection of hyperparameters like time window size and architectural factors analysed by FOA.

The optimal number of hidden neurons in each hidden layer is examined by FOA. Primary weights of the network are set as random values, and the network weight is tuned by using a gradient-based "Adam" optimizer.

The figure 2 shows a flow chart of proposed work. Set $m$ as LSTM's hyperparameters in attractiveness firefly and the exact prediction is done by LSTM as a criterion for calculating firefly positions. Next process is to search the flies iteratively. 
Step1: Initialize the current position of fireflies and the hyperparameters LSTM to be optimized with the estimation of the upper and lower limit.

Step2: Substitute the LSTM hyperparameters corresponding to the every firefly position.

Step3: Divide dataset into train-set and test-set by updating recent data then predict the train-set next Sequence.

Step4: Compare the predicted value and test-set data

Step 5: Evaluate the error between the predicted value and test-set data using (11)

Step6: Evaluate the fitness function by brightening flashes of a firefly, and every firefly position changes using Equation (4).

Step7: Repeat Step 3-6 until satisfied.

Step 8: Result of optimized prediction is the hyperparameter position of firefly by LSTM

\section{Result and Discussions}

For the experiment, the data sets have been downloaded from METEONORM. METEONORM utilizes the normal qualities from the period 1961-1990 for various climate stations. The data set is separated into a training set and holdout sets. For prediction, pressure and temperature sensor values are used as input variables. The output of prediction models is detecting hazards or up normal event in HVAC systems

In this work the performance parameters of Root Mean Square Error (RMSE), Mean Absolute Error (MAE), and Mean Absolute Percentage Error (MAPE) used to find the error between actual and forecasted data. For air, comparison proposed prediction results compared with standard models of SVR (Support Vector Machine based Regression), and LSTM

RMSE characterizes the difference between the predicted value and the actual value. It is mostly used for finding the values at a different time interval. The prediction is more out of true when the value is high.

$$
R M S E=\sqrt{\frac{\sum_{i=0}^{n-1}\left(y_{i}-f_{i}\right)^{2}}{n}}
$$

The mean absolute error (MAE) is a quantity of errors between paired observations. The average error rate is,

$$
M A E=\frac{\sum_{i=0}^{n-1}\left(y_{i}-f_{i}\right)}{n}
$$

A statistical degree of the accurateness of a forecast model is the mean absolute percentage error (MAPE). It is estimated for each period by dividing the subtracted from the actual values to the actual values

$$
M A P E=\frac{\sum_{i=0}^{n-1}\left(y_{i}-f_{i}\right)}{n y_{i}} X 100
$$

Table 2. Performance measure.

\begin{tabular}{|l|l|l|l|}
\hline Method & MSE & MAE & MAPE \\
\hline SVR & 198.5 & 13.3 & 1.30 \\
\hline LSTM & 179 & 11 & 0.9 \\
\hline FOLSTM & 174.99 & 10.18 & 0.86 \\
\hline
\end{tabular}

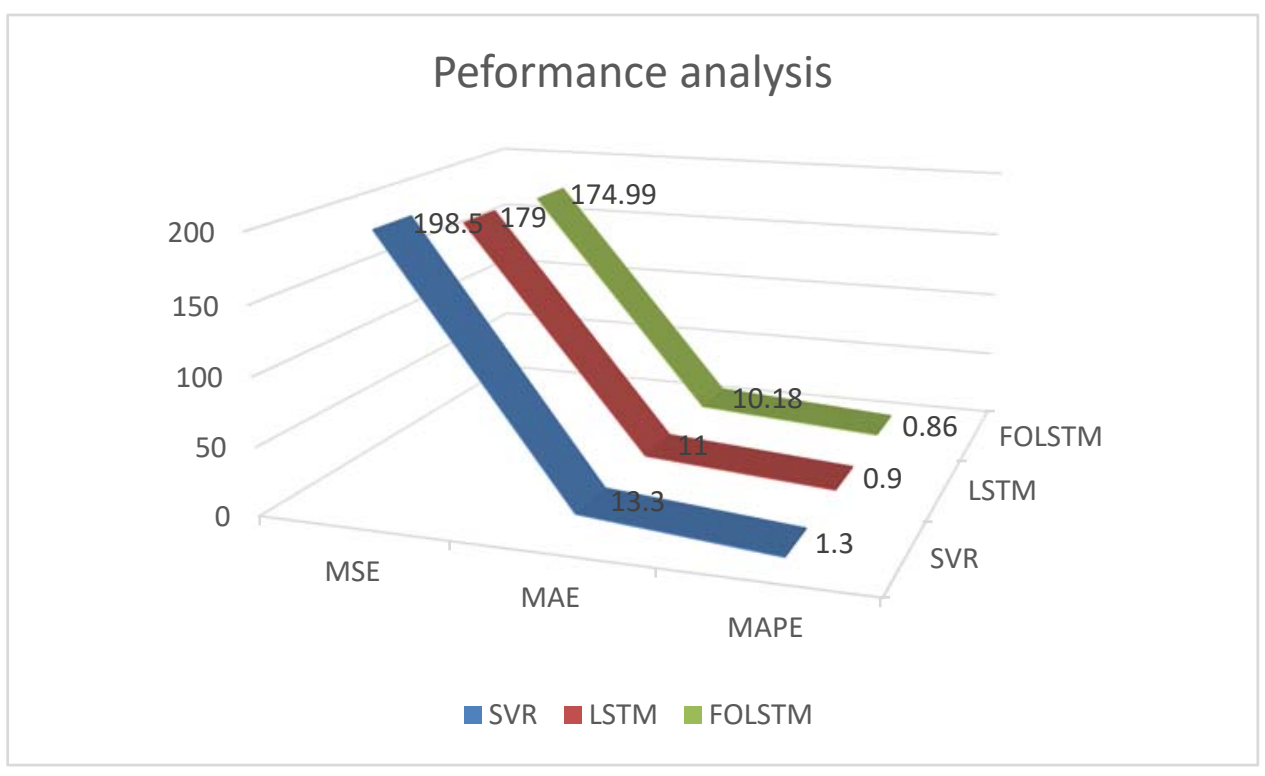

Figure 3 Performance analysis 
The comparison result of proposed FOLSTM is shown in Table 1 and Figure 2, where the proposed FOLSTM is proved that it was much better than the Conventional method of SVR and LSTM respectively. In the MSE benchmark, the SVR has its value as 198.5 and LSTM has its value as 179 is 209.45, while the predicted MSE for proposed FOLSTM is 174.99 which enhances the performances by $13.11 \%$ than existing. Similarly the MAE and MAPE benchmark model of proposed FOLSTM is better than the SVR and LSTM respectively.

\section{Conclusion:}

In this paper, By IoT, enable the improvement of Predictive Maintenance. IoT based systems need to be enhanced with data science abilities, to attain the best objective of digitalization, which is supportive for decision making to optimally act on the physical systems. In this work, a hazard prediction model for Retail shops based on optimized LSTM networks was established. The experimental validation on data set shows that the proposed prediction model has better MAPE, MSE and MAE result performance. These entire outcomes show that optimized LSTM method can be an effective method for hazards and condition forecasting in retail shop systems.

\section{References}

[1] Ben-Nakhi, A. E., \& Mahmoud, M. A. (2004). Cooling load prediction for buildings using general regression neural networks. Energy Conversion and Management, 45(13-14), 2127-2141.

[2] Kwok, S. S., Yuen, R. K., \& Lee, E. W. (2011). An intelligent approach to assessing the effect of building occupancy on building cooling load prediction. Building and Environment, 46(8), 1681-1690.

[3] Nguyen, T. A., \& Aiello, M. (2013). Energy intelligent buildings based on user activity: A survey. Energy and buildings, 56, $244-257$.

[4] Li, Q., Meng, Q., Cai, J., Yoshino, H., \& Mochida, A. (2009). Applying support vector machine to predict hourly cooling load in the building. Applied Energy, 86(10), 2249-2256.

[5] Xu, C., Chen, H., Wang, J., Guo, Y., \& Yuan, Y. (2019). Improving prediction performance for indoor temperature in public buildings based on a novel deep learning method. Building and Environment, 148, 128-135.

[6] Doukas, H., Patlitzianas, K. D., Iatropoulos, K., \& Psarras, J. (2007). Intelligent building energy management system using rule sets. Building and environment, 42(10), 3562-3569.

[7] Alawadi, S., Mera, D., Fernández-Delgado, M., Alkhabbas, F., Olsson, C. M., \& Davidsson, P. (2020). A comparison of machine learning algorithms for forecasting indoor temperature in smart buildings. Energy Systems, 1-17.

[8] Dezfouli, M. M. S., Yazid, M. Z. A., Zakaria, A., Ahmed, S. F., Ali, A., \& Moghimi, S. (2018, May). Application of high efficiency motors in HVAC system for energy saving purpose. In 2018 IEEE International Conference on Innovative Research and Development (ICIRD) (pp. 1-5). IEEE.

[9] Chang, L., Wang, H., \& Wang, L. (2017, September). Cloud-Based parallel implementation of an intelligent classification algorithm for fault detection and diagnosis of HVAC systems. In 2017 International Smart Cities Conference (ISC2) (pp. 1-6). IEEE.

[10] RR Karthikeyan, Dr. B Raghu, Design of Smart Facility Management System, International Journal of Engineering Trends and Technology Volume 68 Issue 9, 167-175, November 2020.

[11] Preglej, A., Rehrl, J., Schwingshackl, D., Steiner, I., Horn, M., \& Škrjanc, I. (2014). Energy-efficient fuzzy model-based multivariable predictive control of a HVAC system. Energy and Buildings, 82, 520-533.

[12] Beghi, A., Cecchinato, L., Rampazzo, M., \& Simmini, F. (2010, December). Load forecasting for the efficient energy management of HVAC systems. In 2010 IEEE International Conference on Sustainable Energy Technologies (ICSET) (pp. 1-6). IEEE.

[13] Johari, N. F., Zain, A. M., Mustaffa, N. H., \& Udin, A. (2017). Machining parameters optimization using hybrid firefly algorithm and particle swarm optimization. In Journal of Physics: Conference Series (Vol. 892, p. 012005).

[14] Blum, C., \& Roli, A. (2003). Metaheuristics in combinatorial optimization: Overview and conceptual comparison. ACM computing surveys (CSUR), 35(3), 268-308.

[15] Xiao, Y., \& Yin, Y. (2019). Hybrid LSTM neural network for short-term traffic flow prediction. Information, $10(3), 105$.

[16] Poppi S. (2010). Development of commercial refrigeration systems. Master Thesis University of Padova

[17] Remund J. (2005). Software METEONORM HYPERLINK http://www.meteonorm.com/pages/en/meteonorm.php.

[18] RR Karthikeyan, Dr. B Raghu, Design of Event Management System for Smart Retail Stores with IoT Edge, International Journal of Engineering Trends and Technology Volume 68 Issue 11, 81-88, November 2020. 